\title{
Una mirada rápida a la investigación ambiental contemporánea en Panamá
}

\section{Luis Wong Vega ${ }^{1, *}$}

${ }^{1}$ Asesor técnico, Dirección de I+D, Secretaría Nacional de Ciencia, Tecnología e Innovación, Panamá, República de Panamá.

*Autor para correspondencia. Email: lwong@senacyt.gob.pa

Recibido: 14 de noviembre de 2014

Aceptado: 5 de diciembre de 2014

\begin{abstract}
Contemporary environmental research is a crucial element within modern environmental governance. It provides direct knowledge on the state of human habitats and natural areas as well as on the resources of our biological and geographic heritage. It provides information to determine policies and actions for its sustainable utilization and preservation. In Panama, the nature end impact of environmental research need a closer examination, as well as changes directed to overcome specific handicaps (mostly of conceptual, institutional and political nature). This paper takes a first look at very important aspect of our environmental culture and state specific suggestions on the matter of its development.
\end{abstract}

Keywords: Research, Environment, Indicators, ANAM, SENACYT, Panamá.

\section{Resumen}

La investigación ambiental contemporánea es un elemento crucial dentro de la gobernanza ambiental moderna. Proporciona un conocimiento directo sobre el estado de los hábitats humanos y áreas naturales, así como sobre los recursos de nuestro patrimonio biológico y geográfico. Aporta información necesaria para determinar las políticas y acciones dirigidas a su utilización sostenible y su conservación. En Panamá, la naturaleza y el impacto final de la investigación ambiental necesita de un examen más detallado, así de como los cambios dirigidos a superar las dificultades específicas (en su mayoría de naturaleza conceptual, institucional y política) que le afectan. En este trabajo se da un primer repaso a aspectos muy importantes de este problema y se añaden sugerencias específicas para su desarrollo.

Palabras clave: Investigación; Ambiente; Indicadores; ANAM; SENACYT; Panamá. 
La investigación y el desarrollo son los medios a través de los cuales el conocimiento se emplea, se reproduce y se proyecta a escala de la sociedad. La investigación permite aprovechar, efectiva y productivamente, el talento y el potencial profesional de nuestros profesionales formados dentro de diversas disciplinas pertinentes al ambiente, especialmente aquellos que se dedican, por vía de la investigación, a generar nuevo conocimiento, nueva información, nuevo material para asimilar e incorporar en procesos formativos, con base en el estudio de las particularidades del ambiente y de nuestro patrimonio natural.

La investigación ambiental moderna es cada vez un ámbito más importante dentro de las agendas de desarrollo socioeconómico sustentable. Tal como detalla el Ministerio de Ambiente de Colombia “...El espectro de la investigación en temas ambientales es en sí amplio y bastante complejo, por lo que se ha visto necesario definir el concepto de investigación ambiental como aquella que se ocupa del estudio del entorno físico-biótico, de su relación con la estructura sociocultural, y de las dinámicas que tal relación conlleva. En tal sentido, la investigación y el conocimiento del ambiente son parte integral de la vida cotidiana de las poblaciones, y deben ser la base para la construcción de las relaciones armónicas con sus respectivos entornos...." (1).

En Panamá, la investigación sobre el tema ambiental y de los recursos naturales no es tratada en forma correcta y por ello, se entiende su relativo nivel de atraso en diferentes niveles (estado del arte, identificación y organización de actores, inversión de recursos, formación de personal especializado, resultados concretos de su acción en términos de proyectos de investigación y publicaciones, programas y acciones de transferencia tecnológica y extensión, etc.).

Desde hace años y como una tendencia reiterada, dentro del Plan Estratégico Nacional de Ciencia y Tecnología (PENCYT), preparado quinquenalmente por la Secretaría Nacional de Ciencia, Tecnología e Innovación (SENACYT) la materia ambiental y de los recursos naturales es tratada tangencialmente, dentro de dos programas sectoriales para el desarrollo científico, tecnológico y de innovación: el de las Biociencias y Ciencias de la Salud y el del Sector Agropecuario, Acuícola, Pesquero y Forestal. El énfasis, dentro de este Plan, se centra en los aspectos de la biodiversidad y en el tema forestal, básicamente.

Incluso dentro de la próxima versión del PENCYT 2014-2019, el tema ambiental es tratado como temática transversal, inmerso en como otro componente más dentro de las cinco áreas estratégicas que lo componen: CTI para el Desarrollo Sostenible; CTI para el Desarrollo Inclusivo; Innovación para la Competitividad; Desarrollo de las Ciencias y Capacidades Científicas-Tecnológicas, y Gobernanza del Sistema SNCTII (2).

\section{Los recursos para la investigación ambiental.}

La investigación ambiental contemporánea y de bien nivel necesita disponer de una serie de condiciones diversas, para poder conducirse y rendir resultados de calidad. Particularmente en el caso de las 
Invest. pens. crit.

Vol. 2, No. 5, septiembre-diciembre 2014.

pp. $80-88$

ciencias ambientales, las condiciones técnico-materiales son, con seguridad, las más difíciles de satisfacer. La adquisición de los equipos y materiales básicos para el trabajo en el laboratorio y en el campo (desde ambientes silvestres hasta núcleos urbanos densamente poblados), el acceso a medios bibliográficos suficientes y actualizados, así como la disposición de recurso humano auxiliar, representan condiciones sine qua non para desarrollar trabajos de investigación, sobre aspectos referidos al ambiente y a nuestros recursos naturales, considerados fundamentales para el desarrollo socioeconómico moderno.

Por otro lado y pese a avances significativos gracias a los programas de financiación vía subsidios competitivos, de la Secretaría Nacional de Ciencia y Tecnología (SENACYT), el acceso a tales recursos ha sido tradicionalmente limitado para los investigadores ambientales.

Por ejemplo: No obstante los recursos dispuestos por el Estado panameño para subsidiar proyectos de investigación en el último decenio (Figura 1a) la carencia de un sistema nacional de adjudicación de subsidios a proyectos de investigación ambiental específicamente, la falta de movilidad (y la eventual desactualización) de los investigadores y dificultades materiales y presupuestarias reales, han causado un estancamiento en la competitividad de los investigadores ambientales nacionales ( $y$, por ende, en la calidad de su trabajo), en general. En la referida figura, se desprende que de los magros recursos de inversión en ciencia, el tema "Biodiversidad y Ecología" ha recibido el 15.23\% (B/. 2,894,952.82) de los fondos adjudicados, lo cual se desglosa en $15.92 \%$ de los proyectos subsidiados por SENACY'T hasta 2014.

Figura 1a. Adjudicación de subsidios en SENACYT hasta 2014

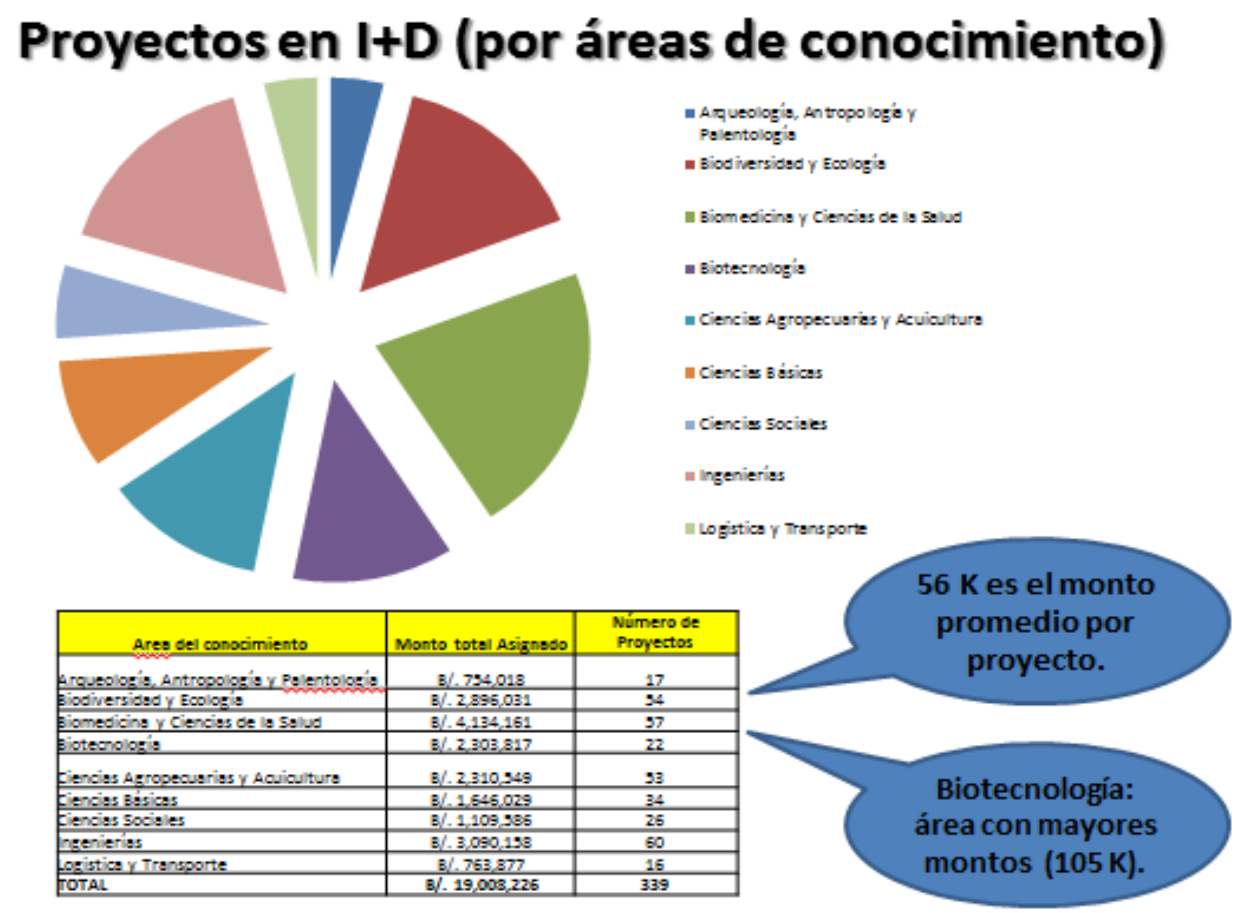


Fuente: Infografía de la Dirección de Investigación y Desarrollo de SENACYT.

Pero si vamos al desglose fino (Figura 1b) de la adjudicación final de estos (ya de por sî) limitados fondos para la investigación ambiental, vemos que una institución extranjera (el Instituto Smithsonian de Investigaciones Tropicales o STRI), ha recibido el $80.47 \%$ de dichos recursos.

Figura 1b. Adjudicación de subsidios en SENACYT hasta 2014 por entidad receptora

\section{Datos por entidades de investigación}

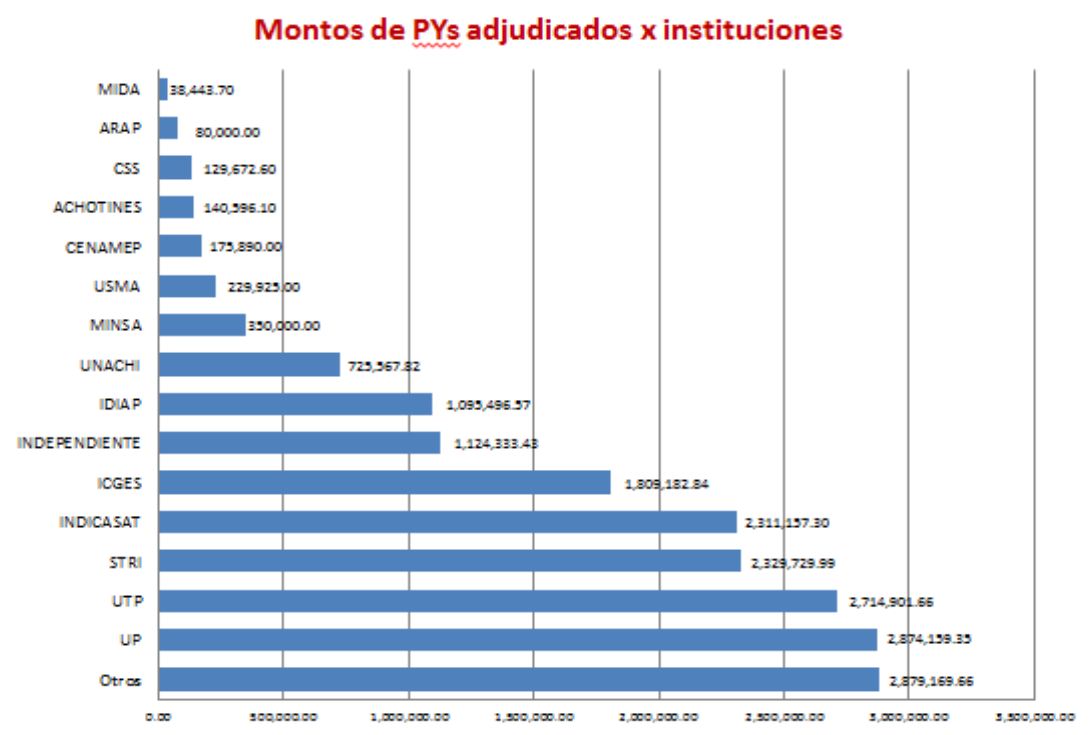

Otros: ACP, ACD, APASAN, BARRILES S.A., CATHALAC, CEFA, CELA, INISA, WWF, PROCOSOL, ULAT, U. OF TULANE, U. DE SAN MARTIN, UDELAS, U. INTERAMERICANA, etc.

Fuente: Infografía de la Dirección de Investigación y Desarrollo de SENACYT.

\section{El impacto de la investigación ambiental actual. Actores y resultados.}

Las indicadores nacionales de productividad científica señalan que la contribución de las ciencias ambientales al aporte panameño al acervo mundial de conocimientos (Figuras 2a y 2b), ha sido y desde muchos años, significativo, no obstante estar sufriendo un declive gradual y sostenido, dentro del grueso de publicaciones indexadas que genera anualmente la comunidad científica del país. 
Invest. pens. crit.

Vol. 2, No. 5, septiembre-diciembre 2014.

pp. $80-88$

Figura 2a. Tipología de las publicaciones científicas de Panamá, según Scimago Journal and Country Rank, 2013

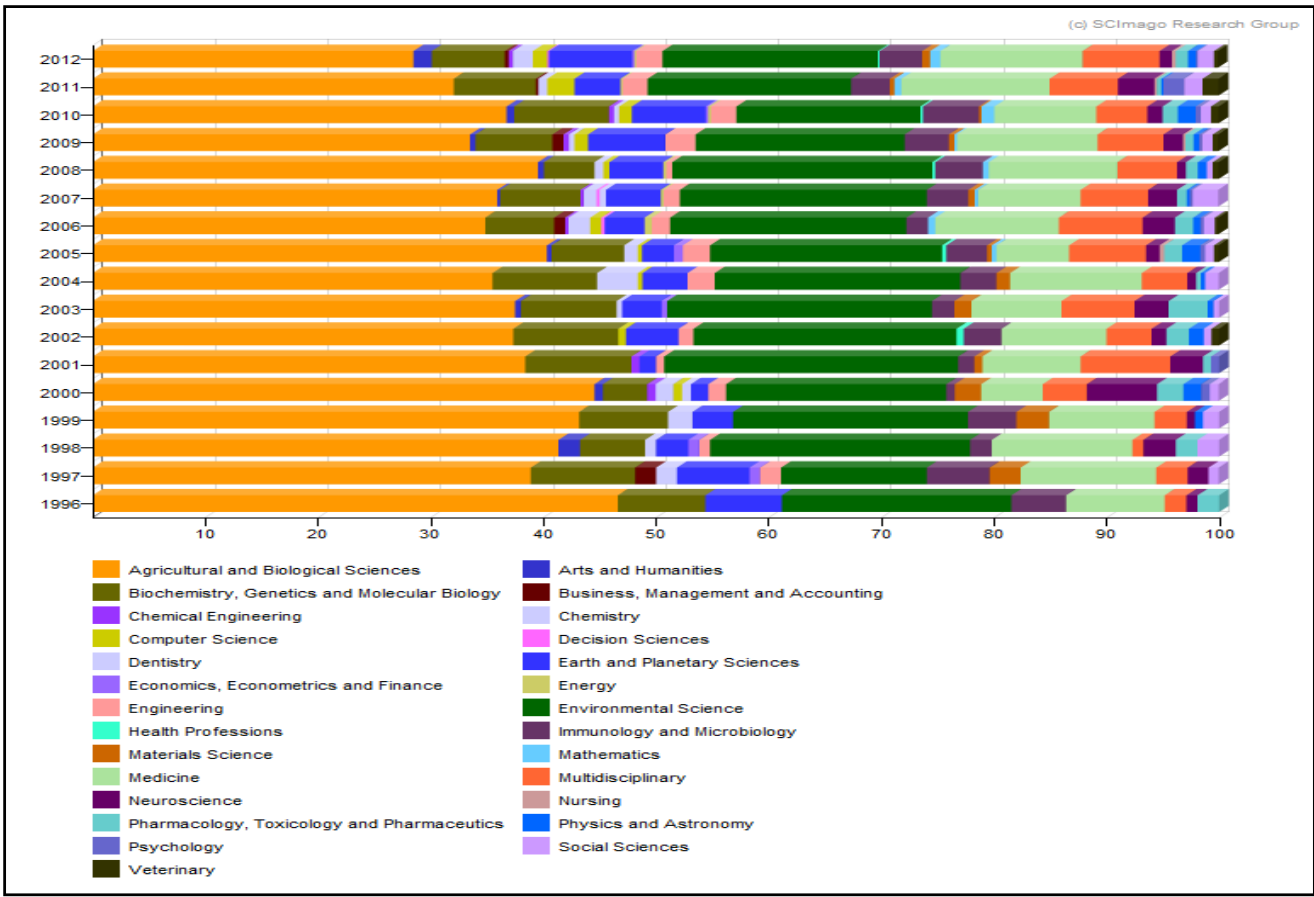

Figura 2b. Tipología de las publicaciones científicas de Panamá, según Scimago Journal and Country Rank, 2013

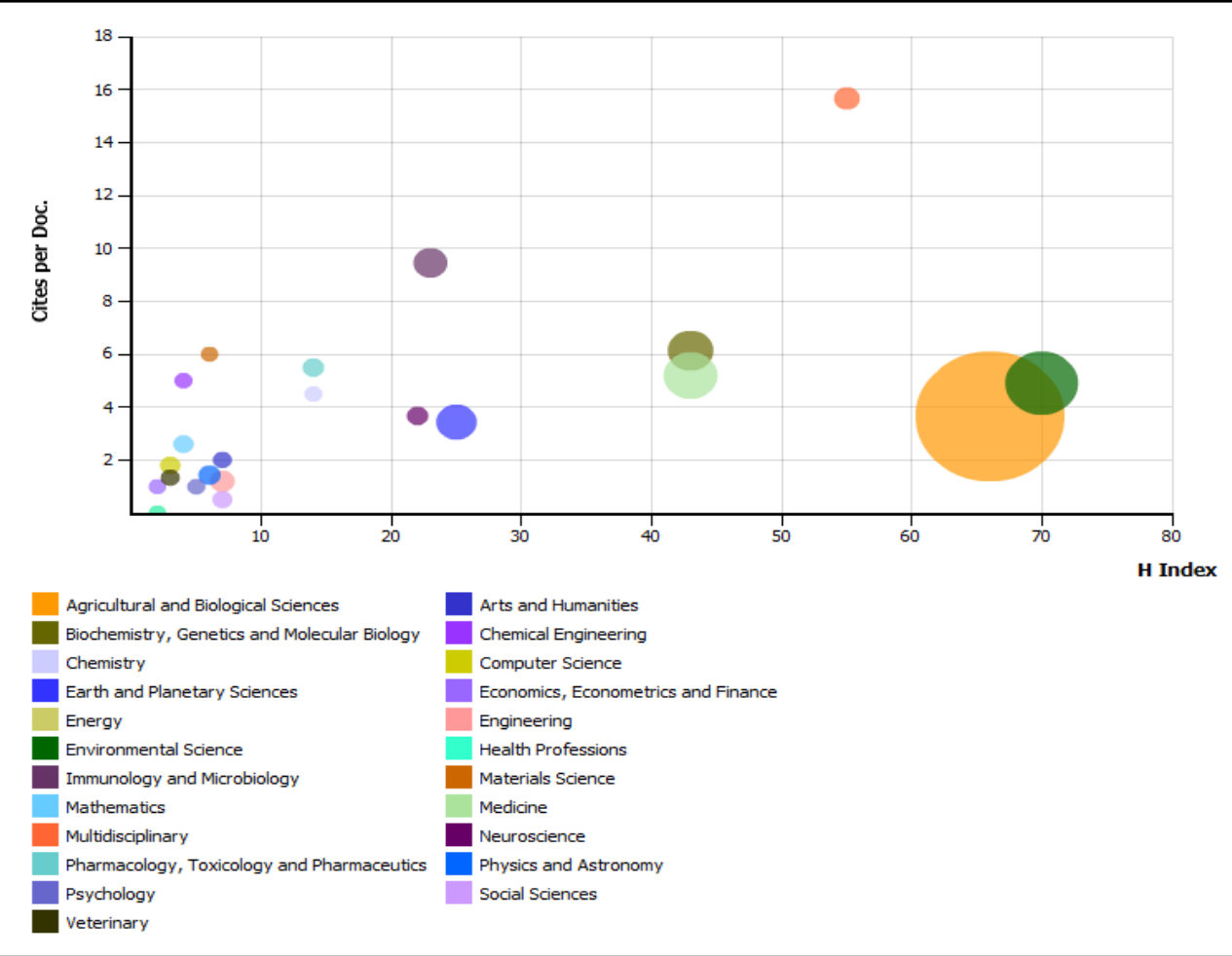


Bases de datos como "Researchgate" muestran cifras comparativas de productividad para las instituciones con competencia directa e indirecta sobre investigación ambiental y la diferencia no puede ser más notable: la preponderancia del Instituto Smithsonian de Investigaciones Tropicales (STRI) en este tema es casi absoluta a nivel local, con un índice de impacto científico significativamente alto: Índice RG: 861.67 (3).

Figura 3. Ranking nacional de instituciones de investigación con base en su productividad científica. Researchgate, julio de 2014

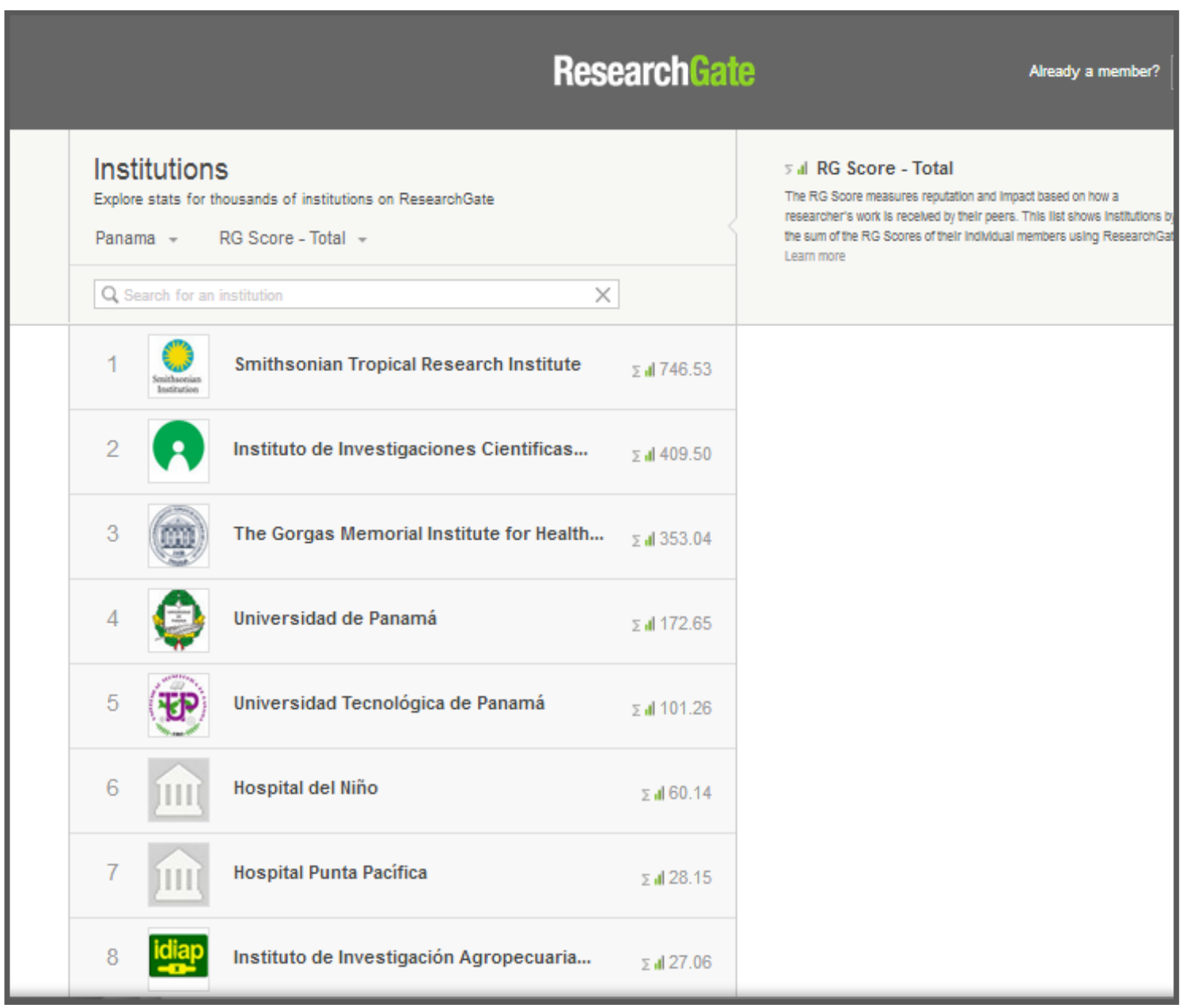

Dentro de esta escalafón y a diciembre de 2014, dentro de las treinta principales organizaciones panameñas generadoras de investigación y publicaciones, ni siquiera figura la ANAM. Ocupando un distante lugar número 28 (de 30) se encuentra el Programa "Corredor Biológico Mesoamericano", con cifras de impacto científico realmente lamentables: valores RG de 0.01 (op.cit).

\section{Por un replanteamiento conceptual del tema, a nivel local. Un nuevo rol para la ANAM.}

Se ha asumido que la investigación ambiental (que muchas veces se confunde con actividades de servicio tecnológico ambiental y hasta con el extensionismo ambiental) debe ser conducida por entidades 
Invest. pens. crit.

Vol. 2, No. 5, septiembre-diciembre 2014.

pp. $80-88$

como las Universidades (por sus departamentos o laboratorios de ciencias ambientales) o por Organismos No Gubernamentales Ambientalistas, así como por entidades investigativas extranjeras (como el STRI).

Bajo este esquema, la investigación ambiental endógena se desarrolla en forma simultánea y desorganizada (muchas veces con duplicidad de esfuerzos), bajo enormes limitaciones financieras y técnicas, sin el amparo de una política clara que fije metas y prioridades, que otorgue incentivos, que promueva publicaciones y acciones de impacto verdadero para el ambiente en el país.

Por otro lado y al igual que en Panamá, la organicidad actual de muchos Ministerios o Agencias Ambientales en Latinoamérica reproduce un patrón, bastante generalizado y común, en donde: “...la estructura y el andamiaje (de la entidad) se dedica casi en su totalidad a actividades administrativas y de gestión (otorgamiento de permisos, licencias y autorizaciones) y a la elaboración de normas y reglamentos ambientales..."(4).

Luego de una revisión exhaustiva de los sitios web de la inmensa mayoría de las agencias ambientales de Latinoamérica y el Caribe, podemos afirmar que esta situación es reiterada y que, conscientes de ello, algunos países ya han comenzado a desarrollar sus propias instancias estatales de investigación ambiental (5).

Curiosamente, en países desarrollados del hemisferio, como Estados Unidos y Canadá, sus agencias ambientales poseen fuertes componentes de investigación propios. En Estados Unidos de América, la Agencia de Protección Ambiental (Environmental Protection Agency o EPA) posee una oficina de Investigación y Desarrollo (Office of Research and Development, ORD) que coordina un plan nacional estratégico con seis líneas mayores de acción nacional y que gestiona un sistema de laboratorios estatales que estudian toda gama de variables ambientales. Para la EPA: “...Science at EPA provides the foundation for credible decision-making to safeguard human health and ecosystems... The Office of Research and Development (ORD) is the scientific research arm of EPA, whose leading-edge research helps provide the solid underpinning of science and technology for the Agency..." (6).

En Canadá, la agencia "Environment Canadá" (o EC), es una fuerte agencia federal con competencias sobre el ambiente y los recursos naturales y una red de laboratorios altamente especializados. EC posee una División de Ciencia y Tecnología, que desarrolla un plan estratégico para las ciencias ambientales canadienses, de naturaleza quinquenal. Para EC: “...Our scientists and engineers provide expert advice, help establish research priorities and criteria to guide funding decisions; they also oversee financial matters, support Environment Canada's participation in interdepartmental program governance, and evaluate the environmental outcomes of funded projects..." (7).

\section{Algunas recomendaciones.}

Creemos que en la naturaleza y atribuciones de la ANAM actual existen falencias importantes que, de ser atendidas efectivamente, pueden ayudar a revertir este cuadro. La oportunidad que abre el eventual 
cambio de condición de la ANAM (8), que pasaría a ser un nuevo ministerio, permitiría enmendar estas carencias y crear condiciones para desarrollar correctamente la investigación sobre nuestro entorno y los recursos de nuestro patrimonio natural, biológico y geográfico.

Una acción efectiva sería la creación de una instancia nueva que atienda específicamente el tema de investigación ambiental, dentro de la estructura de la ANAM actual (o del propuesto Ministerio del Ambiente). Con ella, se buscaría dotar al país con una capacidad propia para liderar la investigación ambiental en el país. Esta unidad técnica estaría a cargo de promover la investigación y de generar conocimiento, asumiendo autónomamente competencias que, en forma limitada, radican hoy en la actual Dirección nacional de Fomento a la Cultura Ambiental.

Igualmente se ocuparía de estructurar una red de centros y laboratorios propios o consorciados en donde se desarrollen capacidades y se atiendan áreas de acción prioritarias en materia ambiental y de conservación de nuestros recursos naturales, así como de ayudar a lograr una efectiva gestión y difusión del conocimiento en materia ambiental, dentro de nuestro país.

Otra acción en el sentido correcto sería la creación de un Fondo Nacional de Investigación Ambiental. Este mecanismo iría destinado a estimular la productividad general de los investigadores ambientales del país, posibilitando la ejecución de nuevos proyectos multidisciplinarios de investigación y/o desarrollo. Buscaría promover una vinculación sinérgica, productiva y permanente entre investigadores ambientales y de los recursos naturales dentro y fuera del país, dentro de una cultura de la investigación basada en la cooperación científica y en unos altos estándares de calidad y rigor. Igualmente, ayudaría a sentar las bases para el establecimiento, desarrollo y fortalecimiento de un Sistema Nacional de Investigación Ambiental y de los Recursos Naturales de Panamá.

\section{Referencias}

1. Política Nacional de Investigación Ambiental, Ministerio del Ambiente, Bogotá, 2001

2. “SENACYT crea su Plan Estratégico 2015-2019 con actores nacionales e internacionales", en el Boletín electrónico de SENACYT, viernes 5 de diciembre de 2014.

3. Researchgate.net. ResearchGate Institutions. 2014 https://www.researchgate.net/institutionsPanama? order $=r g S$ core\&method $=$ total

4. INE, La investigación ambiental para la toma de decisiones, INE, México DF, 2006.

5. Pnuma.org. Ministerios de Medio Ambiente - 2012. http://www.pnuma.org/ministerios/index.htm

6. US EPA. About the Office of Research and Development (ORD). 2014. http://www2.epa.gov/aboutepa/about-office-research-and-development-ord

7. Environment Canada. Environmental Science Technology and the Environment - Science and Technology. 2014. http://www.ec.gc.ca/scitech/default.asp?lang=En\&n=AC4418A5-1 
Invest. pens. crit.

Vol. 2, No. 5, septiembre-diciembre 2014.

pp. $80-88$

8. Proyecto de Ley 025, del 18 de agosto de 2014, "Que crea el Ministerio de Ambiente, modifica la ley 41de 1998, General de Ambiente, y la ley 44 de 2006, que crea la Autoridad de los Recursos Acuáticos de Panamá, y adopta otras disposiciones". 